\title{
Minding the gaps to promote thrombus growth and stability
}

\author{
Lawrence F. Brass, Li Zhu, and Timothy J. Stalker
}

Departments of Medicine and Pharmacology, University of Pennsylvania, Philadelphia, Pennsylvania, USA.

\begin{abstract}
Efforts to understand the role of platelets in hemostasis and thrombosis have largely focused on the earliest events of platelet activation, those that lead to aggregation. Although much remains to be learned about those early events, this Review examines a later series of events: the interactions between platelets that can only occur once aggregation has begun, bringing platelets into close contact with each other, creating a protected environment in the gaps between aggregated platelets, and fostering the continued growth and stability of the hemostatic plug.
\end{abstract}

\section{Introduction}

The mechanisms of platelet activation have grown increasingly familiar. Vascular injury exposes tissue factor within the vessel wall (or allows the local accumulation of circulating tissue factor-containing microparticles), which leads to local generation of thrombin and fibrin. Simultaneously, newly exposed collagen fibrils within the vessel wall become decorated with plasma-derived vWF, circulating platelets adhere long enough to be activated by collagen and thrombin, and a growing mound of platelets is formed as additional circulating platelets are recruited by soluble agonists, including thrombin, $\mathrm{ADP}$, and thromboxane $\mathrm{A}_{2}\left(\mathrm{Tx}_{2}\right)$. Activated platelets stick to each other via bridges formed by the $\alpha_{\mathrm{II}} \beta_{3}$ integrin and multivalent adhesive proteins such as fibrinogen, fibrin, and vWF. They also accelerate thrombin formation. The net result is a hemostatic plug or thrombus composed of activated platelets embedded within a fibrin mesh - a structure that can withstand the shear forces generated by flowing blood in the arterial circulation.

In general terms, this description has withstood the test of time, evolving as new molecules are shown to be participants in or regulators of hemostatic plug formation. Equally important, a number of successful drugs have been developed that target key events in platelet activation with the goal of preventing pathological thrombus formation. These include drugs that block ADP receptors, impair $\mathrm{TxA}_{2}$ synthesis, or prevent the binding of adhesive proteins to activated $\alpha_{\mathrm{IIb}} \beta_{3}$. However, even as it accounts for the initiating events of platelet activation, this model leaves some intriguing questions unanswered. For example, what happens after activated platelets stick to each other? Are interactions between platelets required beyond those mediated by $\alpha_{\mathrm{IIb}} \beta_{3}$ ? What prevents destabilization of the hemostatic plug during the time needed for wound healing to occur? What keeps the integrin engaged with its ligands? What is happening in the gap between activated platelets? How do events on the surface of opposing platelets compare with events on the unopposed surfaces of activated platelets? This Review will address some of these issues, focusing on events within and across the gap between activated platelets and the role that those events play in thrombus growth and stability.

Nonstandard abbreviations used: CD40L, CD40 ligand; ESAM, endothelial cellspecific adhesion molecule; Gas6, growth arrest-specific gene 6; JAM, junctional adhesion molecule; PEAR1, platelet endothelial aggregation receptor 1; SCD40L, soluble CD40L; SLAM, signaling lymphocytic activation molecule; $\mathrm{TxA}_{2}$, thromboxane $\mathrm{A}_{2}$.

Conflict of interest: The authors have declared that no conflict of interest exists.

Citation for this article: J. Clin. Invest. 115:3385-3392 (2005).

doi:10.1172/JCI26869.

\section{Gaps, contacts, and the role of clot retraction}

In contrast to most cells, platelets are not normally in stable contact with each other but develop such contacts once aggregation has begun. Electron micrographs show the close proximity of the plasma membranes of adjacent platelets but do not show adherens or tight junctions such as those formed by epithelial and endothelial cells (1-3). Estimates for the width of the gap between adjacent platelets range from as little as 0 to as much as $50 \mathrm{~nm}$ (4). This short distance between platelets should make it possible for molecules on the surface of one platelet to bind to molecules on an adjacent platelet. This could be a direct interaction, as when one cell adhesion molecule binds to another in trans, or an indirect interaction, such as occurs when multivalent adhesive proteins link activated $\alpha_{\mathrm{IIb}} \beta_{3}$ on adjacent platelets. In either case, these interactions can theoretically provide both an adhesive force and a secondary source of intracellular signaling. Junctional molecules have been detected in platelets, as have some of the molecules associated with junction formation in other types of cells (5). Close contacts between platelets not only allow platelet/platelet interactions to occur but can also limit the diffusion of plasma molecules into the gap between platelets and prevent the escape of platelet activators from within the gap. This might, for example, limit the access of plasmin to embedded fibrin, thereby helping to prevent premature dissolution of the hemostatic plug. It might also foster the accumulation of platelet activators within a protected environment in which higher concentrations can be reached and maintained. Seen in this context, clot retraction, which is dependent on the interaction between actin/myosin complexes and the cytoplasmic domain of $\alpha_{\mathrm{IIb}} \beta_{3}$ as well as the binding of fibrinogen or vWF to the extracellular domain of the integrin, can be viewed as a mechanism for narrowing the gaps between platelets and increasing the local concentration of soluble ligands for platelet receptors (6).

Any list of molecules that participate in contact-dependent and contact-facilitated interactions between platelets is likely to be incomplete, but a number of participants and potential participants have been identified. Those that will be discussed here include integrins and other cell adhesion molecules, direct interactions between receptors and surface-attached ligands, and bioactive molecules that are either secreted or proteolytically shed from the surface of activated platelets (Figure 1 and Table 1). Some of these molecules also play a role in the interaction of platelets with other types of cells, but the focus here will be on platelet/platelet interactions. 


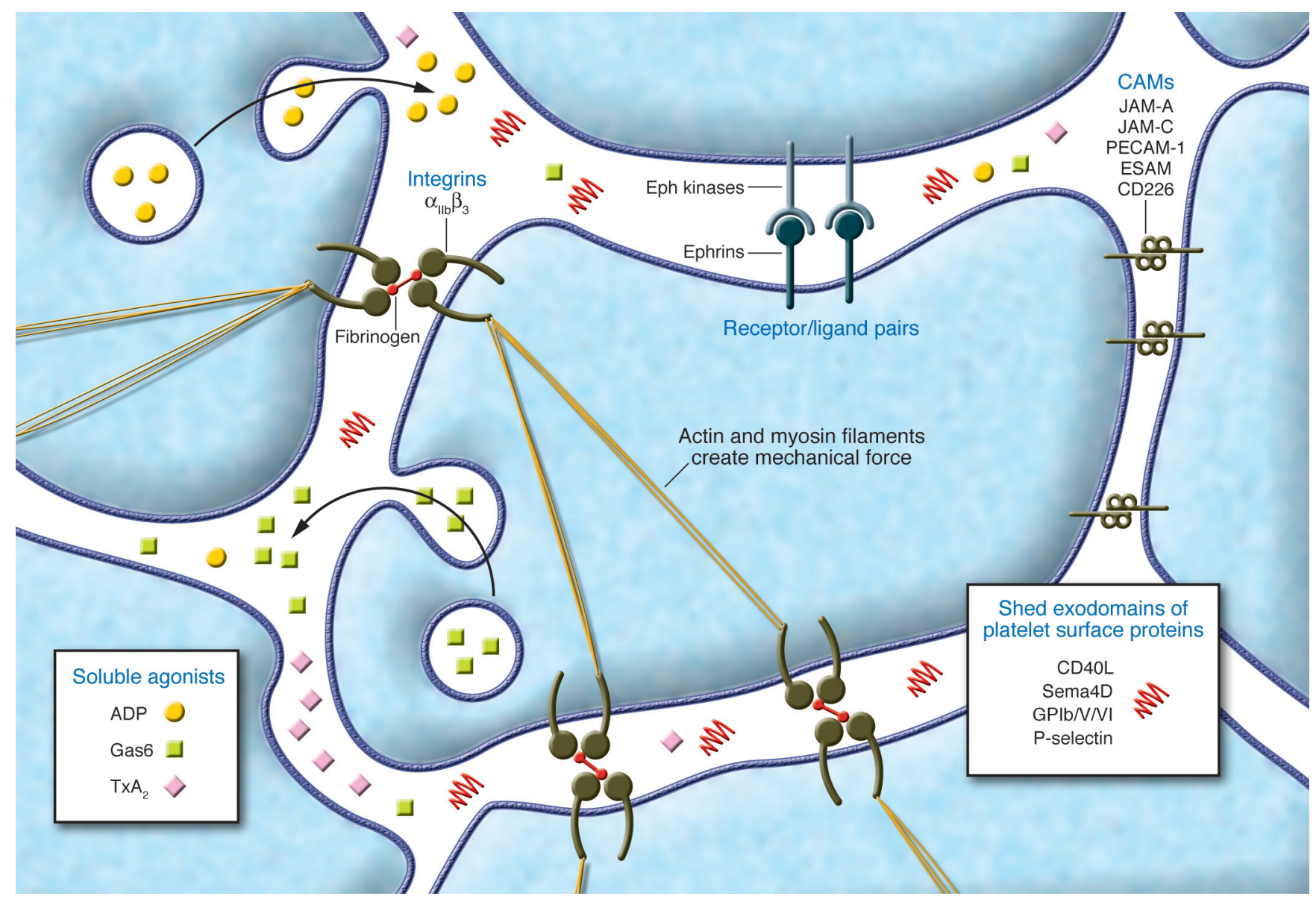

\section{Figure 1}

Events in the gap. The gap between activated platelets in a growing thrombus is small enough for integrins and other cell adhesion molecules (CAMs) to interact and for interactions to occur between receptors such as Eph kinases and their cell surface ligands, known as ephrins. The space between platelets also provides a protected environment in which soluble agonists (ADP and TxA $\mathrm{A}_{2}$ ) and the proteolytically shed exodomains of platelet surface proteins can accumulate. Signaling by ephrins and Eph kinases promotes integrin engagement and outside-in signaling. The mechanical forces generated by the contraction of actin/myosin filaments may compress the space between platelets, improving contacts and increasing the concentration of soluble agonists. The binding of cell adhesion molecules to their partners may limit diffusion into and out of the space between platelets in addition to stabilizing platelet/platelet interactions.

\section{Integrins, adhesion, and outside-in signaling}

Outside-in signaling refers to the intracellular signaling events that occur downstream of activated integrins once ligand binding has occurred. Since this topic has been reviewed by others (7) and is covered in another Review in this series (8), only a few points will be made here. Integrin signaling depends in large part on the formation of protein complexes that link to the integrin cytoplasmic domain. Some of the protein/protein interactions that involve the cytoplasmic domains of $\alpha_{\mathrm{IIb}} \beta_{3}$ help regulate integrin activation; others participate in outside-in signaling and clot retraction. Proteins capable of binding directly to the cytoplasmic domains of $\alpha_{\mathrm{II}} \beta_{3}$ include $\beta_{3}$-endonexin (9), CIB (10), talin (11), myosin (12), Shc (13), and the tyrosine kinases $\operatorname{Src}(14)$ and Syk $(15,16)$. While some of these interactions require the phosphorylation of tyrosine residues Y747 and Y759 in the $\beta 3$ cytoplasmic domain, others do not. Shc, for example, requires Y759 phosphorylation (13). Myosin binding requires phosphorylation of both Y747 and Y759 (12). Fibrinogen binding to the extracellular domain of activated $\alpha_{\mathrm{II}} \beta_{3}$ stimulates a rapid increase in the activity of Src family members and Syk. Studies of platelets from mice lacking these kinases suggest that these events are required for the initiation of outside-in signaling and for full platelet spreading, irreversible aggregation, and clot retraction (17-20).

The phosphorylation of the $\beta 3$ cytoplasmic domain is an event of particular relevance to this Review. Phosphorylation is mediated by 1 or more Src family members and can require both activation of the integrin and its engagement with an adhesive protein (18, 19). Mutation of $Y 747$ and $Y 759$ in $\beta 3$ to phenylalanine produces mice whose platelets disaggregate and which show reduced clot retraction and a tendency to rebleed from sites on the tail where bleeding times were previously measured (21). Loss of clot retraction is also a hallmark of $\alpha_{\mathrm{IIb}} \beta_{3}$-deficient platelets from patients with Glanzmann thrombasthenia - reflecting the dependence of clot retraction on the interaction of $\alpha_{\mathrm{II}} \beta_{3}$ with extracellular fibrin and with intracellular actin/myosin filaments.

\section{Junctional adhesion molecules in the Ig superfamily}

$J A M-A$ and JAM-C. Junctional adhesion molecules (JAMs) are Ig domain-containing, $\mathrm{Ca}^{++}$-independent cell adhesion molecules. 
Three have been identified thus far: JAM-A (also known as JAM-1 and F11R), JAM-B (JAM-2, VE-JAM), and JAM-C (JAM-3) $(22,23)$. Platelets express JAM-A and JAM-C. JAMs have an extracellular domain with 2 Ig domains, a single transmembrane region, and a short cytoplasmic tail that terminates in a binding site for cytosolic proteins with an appropriate PDZ domain. JAM-A localizes to tight junctions of endothelial and epithelial cells and is also found on monocytes, neutrophils, and lymphocytes. JAM-C has been found on endothelial cells, lymphatic vessels, DCs, and NK cells. JAM-A contributes to cell-cell adhesion by forming trans interactions involving the $\mathrm{N}$-terminal Ig domain. However, JAMs also support heterotypic interactions, in particular integrin binding via their membrane-proximal Ig domain. For example, JAM-A binds $\alpha_{\mathrm{L}} \beta_{2}$ integrin on leukocytes and contributes to leukocyte extravasation across the endothelium (24). JAM-C forms adhesive interactions with JAM-B and binds to leukocyte $\alpha_{M} \beta_{2}$ (25).

Platelet JAM-A was originally described as the antigen for a platelet-activating antibody (26). Subsequent studies showed that the activating effects of the antibody are dependent on activation of platelet Fc $\gamma$ RII receptors, a property shared with mAbs directed against other platelet surface proteins as well (27). However, JAM-A has also been shown to induce platelet adhesion and spreading via interactions with immobilized JAM-A in vitro, suggesting that it might play a role in homotypic platelet/platelet and platelet/ endothelial interactions as well as mediating heterophilic interactions between platelets and leukocytes by binding to $\alpha_{L} \beta_{2}(24,28)$. Similarly, JAM-C was found to bind $\alpha_{M} \beta_{2}$ integrin on leukocytes, suggesting a role for JAM-C in platelet/leukocyte interactions (25). The contribution of JAMs to platelet-platelet adhesion has yet to be determined. Interestingly, the cytoplasmic tail of JAM-A becomes phosphorylated via a PKC-dependent mechanism following platelet aggregation (29), suggesting that JAM-A may have a role in contact-dependent signaling.

ESAM. Endothelial cell-specific adhesion molecule (ESAM) is structurally similar to the JAMs (30) with an extracellular domain consisting of $2 \mathrm{Ig}$ domains and a single transmembrane domain. The cytoplasmic tail of ESAM is longer than any of the JAMs and contains several proline-rich regions, as well as a C-terminal PDZ target domain (30). ESAM was identified as a cell adhesion molecule in endothelial cells (30), but it is also expressed by platelets. In endothelial cells ESAM binds via homotypic interactions $(30,31)$, colocalizes with tight junction proteins (31), and binds to the PDZ domain-containing adaptor protein MAGI-1 (32). ESAM is readily detected on the surface of activated platelets, but not on resting platelets, suggesting that it may be located on an intracellular structure, such as the membranes of $\alpha$-granules (31). Its function on platelets is unknown.

CD226. CD226, or DNAM-1, is a fourth Ig superfamily adhesion molecule on the surface of platelets (33). CD226 consists of an extracellular domain with $2 \mathrm{Ig}$ domains, a single transmembrane domain, and a cytoplasmic tail (34). CD226-mediated adhesion of NK cells is dependent on phosphorylation of Ser329 within the CD226 cytoplasmic tail by PKC (35). Cross-linking of CD226 on NK cells results in tyrosine phosphorylation of the cytoplasmic domain and increased cytolytic activity, indicating a possible role in signal transduction. In addition to an association with the integrin $\alpha_{L} \beta_{2}$, a recent study identified the polio virus receptor (PVR; $\mathrm{CD} 155)$ and the adherens junction protein nectin-2 (36) as potential ligands for CD226. CD226 has been shown to participate in the binding of activated platelets to endothelial cells in vitro (34).
Endothelial cells express nectin-2, so it is possible that it serves as a ligand for platelet CD226 as well. It is unknown whether platelets express nectins, or whether CD226 also mediates platelet-platelet adhesion. However, CD226-mediated adhesion and CD226 tyrosine phosphorylation are dependent on platelet activation (34), again suggesting the possibility that CD226 participates in platelet signal transduction pathways in the late stages of platelet activation and thrombus formation.

PECAM-1. Platelet-endothelial cell adhesion molecule (PECAM-1; CD31) is known for its high level of expression on endothelial cells, where it accumulates at junctions between cells. However, as its name suggests, PECAM- 1 is also expressed on the surface of resting and activated platelets. PECAM- 1 is a type 1 transmembrane protein with 6 extracellular Ig domains and an extended cytoplasmic domain of approximately 118 residues (reviewed in ref. 37). The most membrane-distal Ig domain can support homotypic interactions in trans. The C-terminus contains phosphorylatable tyrosine residues that represent tandem ITIM domains capable of binding the tyrosine phosphatases SHP-2 and, possibly, SHP-1 (38). Loss of PECAM-1 expression in mice increases responsiveness to collagen via the GPVI collagen receptor, consistent with a model in which platelet activation leads to tyrosine phosphorylation of PECAM-1, allowing SHP-1/2 to bind and bringing the phosphatase near its substrates, including the Fc $\gamma$ chain partner of GPVI $(39,40)$. This may normally provide a braking effect on collagen-induced signaling and prevent unwarranted platelet activation, but how this fits within the context of contact-induced signaling once platelets begin to aggregate remains to be clarified.

SLAM and CD84. Signaling lymphocytic activation molecule (SLAM; CD150) and CD84 are members of the CD2 family of homophilic adhesion molecules that have been studied extensively in lymphocytes, but have now been shown to be expressed in platelets as well (41-43). The members of the family are type 1 membrane glycoproteins in the Ig superfamily. Notable differences among them are in the cytoplasmic domain, which supports binding interactions with a variety of adaptor/partner proteins. SLAM and CD84 are expressed on the surface of resting as well as activated platelets and become tyrosine phosphorylated during platelet activation, but only if aggregation is allowed to occur (41). Immobilized CD84 causes platelet activation in vitro. Mice that lack SLAM have a defect in platelet aggregation in response to collagen or a PAR4-activating peptide, but a normal response to ADP and a normal bleeding time. In a mesenteric vascular injury model, female $S L A M^{-/-}$mice showed a marked decrease in platelet accumulation. Male mice were normal (41). The presence of SLAM, CD84, and 2 of their known adaptor proteins (SAP and EAT-2) in platelets provides a novel mechanism by which close contacts between platelets can support thrombus stability.

\section{Contact-dependent signaling}

Eph kinases and ephrins. Direct contacts between platelets promote signaling by more than 1 mechanism. In addition to signaling events that occur downstream from cell adhesion molecules, there are receptors that interact in trans with cell surface ligands. This type of interaction is illustrated by Eph kinases and their membrane-bound ligands, known as ephrins. Eph kinases are a large family of receptor tyrosine kinases with an extracellular ligand-binding domain and an intracellular kinase domain and PDZ target domain. Like signaling by other receptor tyrosine kinases, signaling by the Eph kinases is promoted by the clus- 


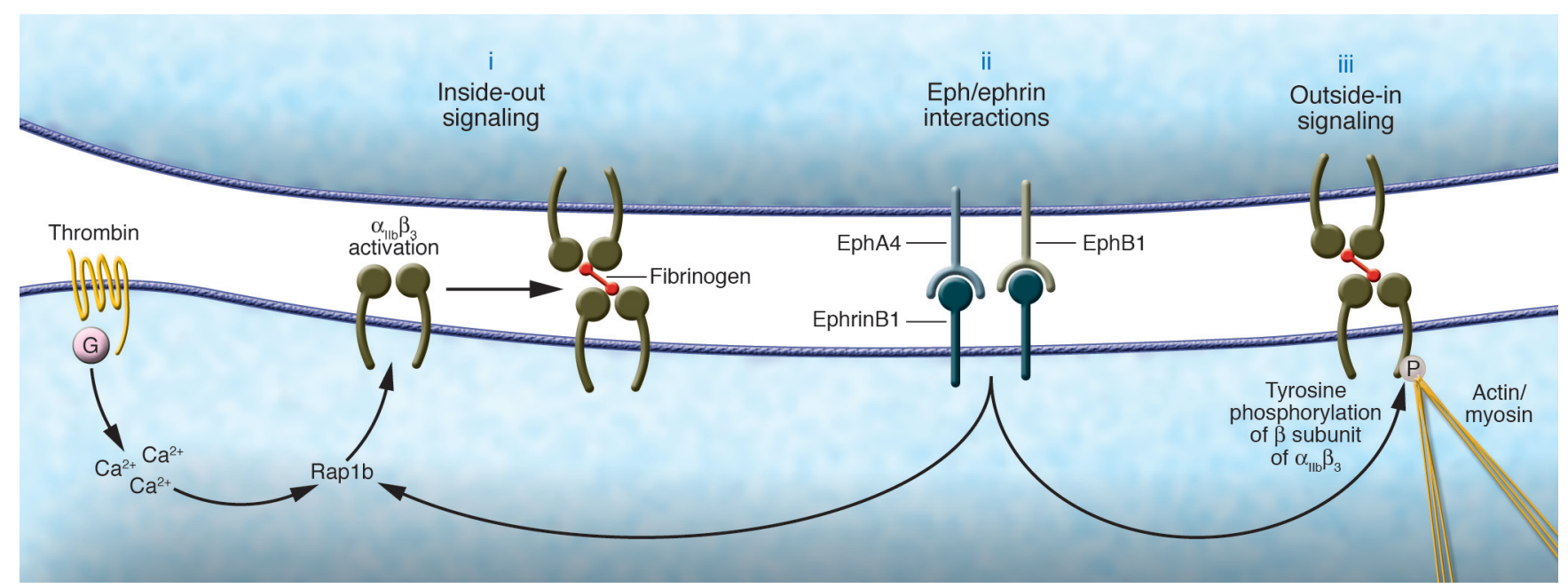

Figure 2

Eph/ephrin interactions between platelets. Agonists such as thrombin, ADP, and TxA 2 are ligands for G protein-coupled receptors, causing a rapid increase in the cytosolic $\mathrm{Ca}^{2+}$ concentration and producing the inside-out signaling events that lead to integrin $\alpha_{\| b} \beta_{3}$ activation (i). The increasingly stable contacts between platelets permit ephrinB1 to bind to EphA4 and EphB1. As discussed in the text, Eph/ephrin interactions support inside-out signaling by activating Rap1b (ii) and also promote outside-in signaling by promoting the tyrosine phosphorylation of the $\beta$ subunit of $\alpha_{\| 1 \mathrm{~b}} \beta_{3}$ (iii).

tering and transphosphorylation induced by ligand binding. The ligands for Eph kinases are cell surface proteins known as ephrins that fall into 2 groups depending on whether they have a glycophosphatidylinositol anchor (the ephrin A family) or a transmembrane domain (the ephrin B family). In turn, Eph kinases are divided into 2 groups by ligand preference. For the most part, A ligands bind to A kinases and B ligands bind to B kinases, although exceptions do exist $(44,45)$. The cytoplasmic domains of the ephrin B family members are highly related. Each is composed of 90-100 residues with 5 conserved tyrosines, at least 3 of which can become phosphorylated (46), and a C-terminus that can serve as a binding site for cytoplasmic proteins with appropriate PDZ domains (47-50).

Eph kinases and ephrins are best known for their role in neuronal organization and brain development (51-53), and as markers distinguishing arteries from veins during vasculogenesis (54). Ephrins can also serve as viral entry receptors (55). Eph/ephrin interactions are particularly relevant to contact-dependent signaling because the binding of an ephrin to an Eph kinase triggers signaling in both the receptor-expressing cell and the ligand-expressing cell (47, 48, 56-60). These events can be dependent on the tyrosine phosphorylation of Eph and ephrin, but phosphorylation-independent interactions have also been described, including those mediated by the PDZ target domains (47-49, 57, 61-65) and the sterile $\alpha$ motif domains (66-69). Taken together, these binding domains make possible the regulated formation of large signaling complexes around clustered Eph kinases and ephrins $(45,51,70,71)$.

Human platelets express 2 Eph kinases, EphA4 and EphB1, and at least 1 ligand, ephrinB1, that can bind to both (72). Forced clustering of either EphA4 or ephrinB1 causes platelets to adhere to immobilized fibrinogen. Clustering of ephrinB1 also causes the activation of Rap1B, a known intermediate in integrin activation in platelets $(73,74)$, and promotes platelet aggregation initiated by suboptimal concentrations of agonists $(72,75)$. During platelet activation the signaling complexes that form with EphA4 include the Src family members Lyn and Fyn (72). Blockade of Eph/ephrin interactions leads to reversible platelet aggregation at low agonist concentrations and limits the growth of platelet thrombi on collagen-coated surfaces under arterial flow conditions $(72,76)$. It also impairs phosphorylation of the $\beta 3$ cytoplasmic domain, inhibiting the association of myosin with $\alpha_{\mathrm{IIb}} \beta_{3}$ and, as a result, impairing clot retraction (76). EphA4 is constitutively associated with $\alpha_{\mathrm{II}} \beta_{3}$ in both resting and activated platelets and colocalizes with the integrin at sites of contact between aggregated platelets (76). Collectively, these observations suggest a model in which the onset of aggregation brings platelets into close proximity and allows ephrinB1 to bind to EphA4 and EphB1. Signals downstream of both the receptors and the kinases then promote further integrin activation (in part by activating Rap1b) and integrin signaling (in part by promoting $\beta 3$ phosphorylation). In turn, these events promote thrombus growth and stability (Figure 2).

PEAR1. Platelet endothelial aggregation receptor 1 (PEAR1) is a newly described molecule expressed on platelets and endothelial cells (77). It is a 140 - to $150-\mathrm{kDa}$ type 1 transmembrane protein with 15 EGF repeats that is equally expressed on the surface of resting and activated platelets and has, as yet, no known ligand. Platelet activation leads to the phosphorylation of tyrosine and serine residues within the PEAR1 cytoplasmic domain and supports association with Shc. Phosphorylation in activated platelets can be inhibited by prevention of aggregation, suggesting that PEAR1-dependent events are dependent on contact and not just activation.

\section{Shedding and secreting into the gap}

More than just an empty space, the gap between platelets provides a safe harbor in which platelet-derived molecules can accumulate. In addition to the numerous plasma- and megakaryocyte-derived proteins that are secreted from platelet $\alpha$-granules (78), activated platelets release agonists such as $\mathrm{ADP}$ and $\mathrm{TxA}_{2}$ and presumably continue to do so even after thrombus formation has begun. Platelets also shed surface molecules, including GPIb $\alpha$ (79), GPV (80), GPVI 
Table 1

Molecules that participate in contact-facilitated events in platelets

Integrins and outside-in signaling
Junctional adhesion (and signaling) molecules
JAM-A
JAM-C
ESAM
CD226
PECAM-1
SLAM and CD84
Contact-dependent signaling molecules
EphrinB1 and EphA4/B1
PEAR1
Ligands that are shed or secreted
ADP
TXA 2
CD40L
Sema4D
Gas6

$(81,82)$, and P-selectin (83). Cleavage of each of these proteins can be prevented with inhibitors of metalloproteases, and, in at least 2 cases (GPIb $\alpha$ and GPV), a role for a particular metalloprotease, ADAM17, has been established through studies on platelets from mice that lack it $(79,80)$. The advantage that the platelet derives from shedding surface proteins can be surmised but is not entirely clear. Downregulation of responsiveness to collagen has been proposed as the benefit derived from shedding GPVI (82). Loss of the exodomain of GPIb $\alpha$, which serves as the primary $\mathrm{vWF}$ receptor on platelets, would also impair platelet interactions with collagen, but since cleavage occurs after platelet activation, the impact on thrombus prevention is not readily apparent. It is possible that the reduction in net surface charge that would accompany loss of the negatively charged GPIb $\alpha$ exodomain allows platelets to pack more closely together in a thrombus. It may also expose interaction sites on the remaining extracellular portion of GPIb $\alpha$ that are occluded in the intact molecule, but this remains speculative. In contrast to GPIb and GPVI, where cleavage of the exodomain may predominantly impair function, cleavage of 2 other molecules on the surface of activated platelets, CD40 ligand and sema4D, gives rise to bioactive fragments that can stimulate platelets as well as other nearby cells.

CD40L. CD40 ligand (CD40L; CD154) is a 33-kDa transmembrane protein that is present on the surface of activated platelets, but not resting platelets (84-86). Its appearance on the platelet surface is followed by the gradual release of an $18-\mathrm{kDa}$ exodomain fragment of CD40L (85). Both the surface-bound form and the soluble form of CD40L (sCD40L) are trimers (87). Platelets also express CD40, the $48-\mathrm{kDa}$ transmembrane protein that acts as a receptor for CD40L $(85,88,89)$. Unlike CD40L, CD40 is detectable on the surface of resting as well as activated platelets $(85,88)$. CD40L is a member of the TNF family, and platelet-derived sCD40L or activated, CD40L-expressing platelets can elicit responses from endothelial cells and monocytes that appear to be proatherogenic (84, 85, 90-92). The extracellular portion of CD40L includes a binding domain for CD40 and a KGD (RGD in mice) integrin-recognition sequence. The bind- ing of sCD40L to activated platelets is blocked by mutations in the KGD sequence and by antibodies to $\alpha_{\mathrm{II}} \beta_{3}$. Binding occurs to platelets that lack CD40, but not to platelets that lack $\beta 3$, all of which suggests that SCD $40 \mathrm{~L}$ can bind to activated $\alpha_{\mathrm{IIb}} \beta_{3}$ on platelets and that the KGD sequence is involved (88). Consistent with a role in thrombus formation, $C D 40 L^{-/-}$mice show delayed occlusion following vascular injury and decreased thrombus stability (88). Loss of the gene encoding CD40 has no apparent effect (88). Infusion of SCD40L into $C D 40 \mathrm{~L}^{-/-}$mice reverses the phenotype observed after $\mathrm{FeCl}_{3}$-induced vascular injury, but sCD40L with an altered KGD sequence does not. Notably, platelet aggregation is normal in $C D 40 \mathrm{~L}^{-/-}$platelets, although growth of platelet plugs on collagen-coated surfaces under shear is affected $(88,93)$. All of this suggests that the effects of CD $40 \mathrm{~L}$ on platelets are mediated by $\alpha_{\mathrm{IIb}} \beta_{3}$, not CD 40 , and that platelet behavior in the relatively low-shear conditions present in an aggregometer cuvette may not fully reflect the events that are relevant to thrombus stability.

Sema4D and its receptors. Semaphorins are a large family of structurally related proteins $(94,95)$. Like Eph kinases and ephrins, semaphorins are best known for their role in the CNS, but individual family members have been found elsewhere, including hematopoietic cells. Semaphorins have in common a 500-amino acid residue extracellular "sema" domain that forms a 7-bladed propeller structure (96). Sema4D, or CD100, is a $150-\mathrm{kDa}$ type 1 glycoprotein that forms a disulfide-linked homodimer. Each subunit has an N-terminal sema domain, an Ig domain, a lysine-rich stretch, a transmembrane domain, and a cytoplasmic tail with consensus tyrosine and serine phosphorylation sites whose role is unclear. Sema4D is best known for its role in lymphocyte biology. Soluble sema4D released from activated lymphocytes inhibits immune cell migration, acts as a costimulator for CD40-induced B cell proliferation and Ig production, and induces release of proinflammatory cytokines by monocytes (97-100). Sema4D $D^{-/-}$mice are viable but show defective B cell development, impaired $\mathrm{T}$ cell activation, and blunted immune responses (101). Two receptors have been identified for sema4D: plexin-B1, a high-affinity receptor expressed on endothelial cells $(102,103)$, and CD72, which is expressed on lymphocytes (104-106). Plexin-B1 was recently shown to elicit proangiogenic responses and signaling in endothelial cells $(102,107,108)$.

We have recently identified sema4D on the surface of human and mouse platelets (109). Platelet activation causes progressive loss of intact sema4D and the shedding of its disulfide-linked exodomain. Shedding is sensitive to metalloprotease inhibitors (110). These results suggest that at least some of the sema4D expressed in platelets is initially located on the cell surface and that platelet activation leads to cleavage of the protein by a platelet-associated metalloprotease, releasing a larger fragment and leaving a smaller fragment behind in the membrane. Western blots show that platelets also express CD72 and, therefore, may be capable of responding to as well as releasing sema4D (110). The rate at which sema4D is shed from activated platelets, like the rate of CD40L shedding, lags behind the rate of platelet aggregation. This suggests that at least some of the shed sema4D exodomain should be protected in the gaps between platelets, while the rest is washed downstream where it can bind to endothelial cells.

Gas 6 and its receptors. Growth arrest-specific gene 6 (Gas6) is a $75-\mathrm{kDa}$ secreted protein related to protein S. Like protein S and other proteins whose synthesis requires vitamin $\mathrm{K}$ as a cofactor, Gas 6 contains $\gamma$-carboxylated glutamic acid residues that allow 
it to bind to negatively charged phospholipids in a $\mathrm{Ca}^{++}$-dependent manner (111). Gas6 is expressed in a number of tissues, including vascular smooth muscle, and levels of expression are upregulated following vascular injury. In mouse platelets, Gas 6 is found in the $\alpha$-granules $(112,113)$. Upon activation by thrombin, ADP, or collagen, Gas6 is secreted from rat platelets (112). Gas 6 is a ligand for the related receptor tyrosine kinases Axl, Sky, and Mer $(114,115)$, all of which are expressed on platelets (113). Like other growth factor receptors, Axl family members have been shown to stimulate PI3K and phospholipase $\mathrm{C} \gamma$ (111). A reasonable hypothesis is that secreted Gas 6 could bind to receptors on the platelet surface and cause signaling that would promote platelet plug formation and stability, perhaps while binding to negatively charged phospholipids on adjacent platelets. Consistent with this hypothesis, platelets from $\mathrm{Gas}^{-/-}$ mice were found to have an aberrant response to agonists in which aggregation begins but ends prematurely (113). Furthermore, although the tail bleeding time of the $\mathrm{Gas}^{-/-}$mice was normal, the mice were resistant to thrombosis (113), as are mice lacking any 1 of the 3 Gas 6 receptors. Platelets from the receptor-deleted mice also failed to aggregate normally in response to agonists (116-118). Biochemical studies showed that Gas6 signaling promotes $\beta 3$ phosphorylation and, therefore, clot retraction (117).

Collectively, these results are consistent with a role for secreted Gas6 in promoting integrin outside-in signaling and helping to perpetuate and stabilize platelet plug formation. Most granule secretion occurs after the onset of aggregation, and Gas 6 secreted into the confined spaces between platelets might achieve high local concentrations and cause receptor activation. However, a number of issues remain to be settled before this hypothesis can be fully accepted. In particular, the biology of the Gas6 system needs to be explored further in humans, as most current evidence is from experiments with mice. At least 1 recent study suggests that human platelets do not store and secrete Gas6 (119).

\section{Conclusion}

There is now ample evidence that the signaling events that support platelet activation continue after integrin activation, granule secretion, and platelet aggregation have begun. Some of these events can reasonably be expected to promote the growth and stability of the hemostatic plug, support clot retraction, and help to maintain the plug in place until wound healing is complete or at least well under way. In contrast to the initiating events of platelet activation, these "late" events can take advantage of the close proximity between platelets once aggregation begins and may even extend to the contacts that develop between platelets, endothelial cells, and leukocytes as the hemostatic plug evolves. The models of contact-dependent signaling discussed in this Review are just examples of what will probably turn out to be a more complex process involving additional cell surface molecules whose function remains to be identified. The picture that emerges is that the gaps and contact points between activated platelets in a thrombus are sites of highly relevant activity.

\section{Acknowledgments}

This work was supported by grants from the National Heart, Lung, and Blood Institute (to L.F. Brass) and the American Heart Association (to T.J. Stalker).

Address correspondence to: Lawrence Brass, University of Pennsylvania, Room 915 Biomedical Research Building II, 421 Curie Boulevard, Philadelphia, Pennsylvania 19104, USA. Phone: (215) 573-3540; Fax: (215) 573-2189; E-mail: Brass@mail.med.upenn.edu.
1. Humbert, M., et al. 1996. Ultrastructural studies of platelet aggregates from human subjects receiving clopidogrel and from a patient with an inherited defect of an ADP-dependent pathway of platelet activation. Arterioscler. Thromb. Vasc. Biol. 16:1532-1543.

2. White, J.G. 1972. Interaction of membrane systems in blood platelets. Am. J. Pathol. 66:295-312.

3. White, J.G. 1988. Platelet membrane ultrastructure and its changes during platelet activation. Prog. Clin. Biol. Res. 283:1-32.

4. Skaer, R.J., Emmines, J.P., and Skaer, H.B. 1979. The fine structure of cell contacts in platelet aggregation. J. Ultrastruct. Res. 69:28-42.

5. Elrod, J.W., et al. 2003. Expression of junctional proteins in human platelets. Platelets. 14:247-251.

6. Tschumperlin, D.J., et al. 2004. Mechanotransduction through growth-factor shedding into the extracellular space. Nature. 429:83-86.

7. Shattil, S.J., and Newman, P.J. 2004. Integrins: dynamic scaffolds for adhesion and signaling in platelets. Blood. 104:1606-1615.

8. Bennett, J.S. 2005. Structure and function of the platelet integrin $\alpha_{\mathrm{II}} \beta_{3}$.J. Clin. Invest. 115:3363-3369. doi:10.1172/JCI26989.

9. Shattil, S.J., et al. 1995. $\beta 3$-Endonexin, a novel polypeptide that interacts specifically with the cytoplasmic tail of the integrin $\beta 3$ subunit. J. Cell Biol. 131:807-816.

10. Naik, U.P., Patel, P.M., and Parise, L.V. 1997. Identification of a novel calcium-binding protein that interacts with the integrin alphaIIb cytoplasmic domain. J. Biol. Chem. 272:4651-4654.

11. Calderwood, D.A., et al. 1999. The talin head domain binds to integrin beta subunit cytoplasmic tails and regulates integrin activation. J. Biol. Chem.
274:28071-28074.

12. Jenkins, A.L., et al. 1998. Tyrosine phosphorylation of the beta3 cytoplasmic domain mediates integrin-cytoskeletal interactions. J. Biol. Chem. 273:13878-13885.

13. Cowan, K.J., Law, D.A., and Phillips, D.R. 2000. Identification of Shc as the primary protein binding to the tyrosine-phosphorylated beta3 subunit of alphalIbbeta 3 during outside-in integrin platelet signaling. J. Biol. Chem. 275:36423-36429.

14. Arias-Salgado, E.G., et al. 2003. Src kinase activation by direct interaction with the integrin beta cytoplasmic domain. Proc. Natl. Acad. Sci. U. S. A. 100:13298-13302.

15. Gao, J., Zoller, K.E., Ginsberg, M.H., Brugge, J.S., and Shattil, S.J. 1997. Regulation of the pp72syk protein tyrosine kinase by platelet integrin alphaIIbbeta3. EMBO J. 16:6414-6425.

16. Woodside, D.G., et al. 2001. Activation of Syk protein tyrosine kinase through interaction with integrin beta cytoplasmic domains. Curr. Biol. 11:1799-1804.

17. Payrastre, B., et al. 2000. The integrin alphaIIb/ beta3 in human platelet signal transduction. Biochem. Pharmacol. 60:1069-1074.

18. Philips, D.R., Prasad, K.S.S., Manganello, J., Bao, M., and Nannizzi-Alaimo, L. 2001. Integrin tyrosine phosphorylation in platelet signaling. Curr. Opin. Cell Biol. 13:546-554.

19. Phillips, D.R., Nannizzi-Alamio, L., and Prasad, K.S.S. 2001. $\beta 3$ Tyrosine phosphorylation in $\alpha I I b \beta 3$ (platelet membrane GP IIb-IIIa) outside-in integrin signaling. Thromb. Haemost. 86:246-258.

20. Obergfell, A., et al. 2002. Coordinate interactions of Csk, Src and Syk kinases with alphaIIbbeta3 ini- tiate integrin signaling to the cytoskeleton. J. Cell Biol. 157:265-275.

21. Law, D.A., et al. 1999. Integrin cytoplasmic tyrosine motif is required for outside-in alphaIIbbeta 3 signalling and platelet function. Nature. 401:808-811.

22. Muller, W.A. 2003. Leukocyte-endothelial-cell interactions in leukocyte transmigration and the inflammatory response. Trends Immunol. 24:327-334.

23. Bazzoni, G. 2003. The JAM family of junctional adhesion molecules. Curr. Opin. Cell Biol. 15:525-530.

24. Ostermann, G., Weber, K.S., Zernecke, A., Schroder, A., and Weber, C. 2002. JAM-1 is a ligand of the beta(2) integrin LFA-1 involved in transendothelial migration of leukocytes. Nat. Immunol. 3:151-158.

25. Santoso, S., et al. 2002. The junctional adhesion molecule 3 (JAM-3) on human platelets is a counterreceptor for the leukocyte integrin Mac-1.J. Exp. Med. 196:679-691.

26. Kornecki, E., Walkowiak, B., Naik, U.P., and Ehrlich, Y.H. 1990. Activation of human platelets by a stimulatory monoclonal antibody. J. Biol. Chem. 265:10042-10048.

27. Naik, U.P., Ehrlich, Y.H., and Kornecki, E. 1995. Mechanisms of platelet activation by a stimulatory antibody: cross-linking of a novel platelet receptor for monoclonal antibody F11 with the Fc gamma RII receptor. Biochem. J. 310:155-162.

28. Babinska, A., et al. 2002. F11-receptor (F11R/JAM) mediates platelet adhesion to endothelial cells: role in inflammatory thrombosis. Thromb. Haemost. 88:843-850.

29. Ozaki, H., et al. 2000. Junctional adhesion molecule (JAM) is phosphorylated by protein kinase C upon platelet activation. Biochem. Biophys. Res. Commun. 276:873-878. 
30. Hirata, K., et al. 2001. Cloning of an immunoglobulin family adhesion molecule selectively expressed by endothelial cells. J. Biol. Chem. 276:16223-16231.

31. Nasdala, I., et al. 2002. A transmembrane tight junction protein selectively expressed on endothelial cells and platelets. J. Biol. Chem. 277:16294-16303.

32. Wegmann, F., Ebnet, K., Du Pasquier, L., Vestweber, D., and Butz, S. 2004. Endothelial adhesion molecule ESAM binds directly to the multidomain adaptor MAGI-1 and recruits it to cell contacts. Exp. Cell Res. 300:121-133.

33. Scott,J.L., etal. 1989. Characterization of a novel membrane glycoprotein involved in platelet activation. J. Biol. Chem. 264:13475-13482.

34. Kojima, H., et al. 2003. CD226 mediates platelet and megakaryocytic cell adhesion to vascular endothelial cells. J. Biol. Chem. 278:36748-36753.

35. Shibuya, A., Lanier, L.L., and Phillips, J.H. 1998. Protein kinase $\mathrm{C}$ is involved in the regulation of both signaling and adhesion mediated by DNAX accessory molecule-1 receptor. J. Immunol. 161:1671-1676.

36. Bottino, C., et al. 2003. Identification of PVR (CD155) and Nectin-2 (CD112) as cell surface ligands for the human DNAM-1 (CD226) activating molecule. J. Exp. Med. 198:557-567.

37. Newman, P.J., and Newman, D.K. 2003. Signal transduction pathways mediated by PECAM-1 new roles for an old molecule in platelet and vascular cell biology. Arterioscler. Thromb. Vasc. Biol. 23:953-964.

38. Newman, P.J. 1999. Switched at birth: a new family for PECAM-1. J. Clin. Invest. 103:5-9.

39. Patil, S., Newman, D.K., and Newman, P.J. 2001. Platelet endothelial cell adhesion molecule-1 serves as an inhibitory receptor that modulates platelet responses to collagen. Blood. 97:1727-1732.

40. Jones, K.L., et al. 2001. Platelet endothelial cell adhesion molecule- 1 is a negative regulator of plateletcollagen interactions. Blood. 98:1456-1463.

41. Nanda, N., et al. 2005. Platelet aggregation induces platelet aggregate stability via SLAM family receptor signaling. Blood. doi:10.1182/blood-2005-01-0333.

42. Krause, S.W., Rehli, M., Heinz, S., Ebner, R., and Andreesen, R. 2000. Characterization of MAX.3 antigen, a glycoprotein expressed on mature macrophages, dendritic cells and blood platelets: identity with CD84. Biochem. J. 346:729-736.

43. Martin, M., et al. 2001. CD84 functions as a homophilic adhesion molecule and enhances IFNgamma secretion: adhesion is mediated by Ig-like domain 1. J. Immunol. 167:3668-3676.

44. Gale, N.W., et al. 1996. Eph receptors and ligands comprise two major specificity subclasses and are reciprocally compartmentalized during embryogenesis. Neuron. 17:9-19.

45. Klein, R. 2001. Excitatory Eph receptors and adhesive ephrin ligands. Curr. Opin. Cell Biol. 13:196-203.

46. Kalo, M.S., Yu, H.H., and Pasquale, E.B. 2001. In vivo tyrosine phosphorylation sites of activated ephrin-B1 and EphB2 from neural tissues. J. Biol. Chem. 276:38940-38948.

47. Torres, R., et al. 1998. PDZ proteins bind, cluster, and synaptically colocalize with Eph receptors and their ephrin ligands. Neuron. 21:1453-1463.

48. Lin, D., Gish, G.D., Songyang, Z., and Pawson, T. 1999. The carboxyl terminus of B class ephrins constitutes a PDZ binding motif. J. Biol. Chem. 274:3726-3733.

49. Bruckner, K., et al. 1999. EphrinB ligands recruit GRIP family PDZ adaptor proteins into raft membrane microdomains. Neuron. 22:511-524.

50. Della Rocca, G.J., et al. 1997. Ras-dependent mitogen-activated protein kinase activation by $\mathrm{G}$ protein-coupled receptors: convergence of Gi- and Gqmediated pathways on calcium/calmodulin, Pyk2, and Src kinase. J. Biol. Chem. 272:19125-19132.

51. Kullander, K., and Klein, R. 2002. Mechanisms and function of Eph and ephrin signaling. Nat. Rev. Mol. Cell Biol. 3:475-486.

52. Holmberg, J., and Frisen, J. 2002. Ephrins are not only unattractive. Trends Neurosci. 25:239-243.

53. Depaepe, V., et al. 2005. Ephrin signalling controls brain size by regulating apoptosis of neural progenitors. Nature. 435:1244-1250.

54. Adams, R.H., and Klein, R. 2000. Eph receptors and ephrin ligands: essential mediators of vascular development. Trends Cardiovasc. Med. 10:183-188.

55. Negrete, O.A., et al. 2005. EphrinB2 is the entry receptor for Nipah virus, an emergent deadly paramyxovirus. Nature. 436:401-405.

56. Holland, S.J., et al. 1997. Juxtamembrane tyrosine residues couple the Eph family receptor EphB2/ Nuk to specific $\mathrm{SH} 2$ domain proteins in neuronal cells. EMBO J. 16:3877-3888.

57. Hock, B., et al. 1998. PDZ-domain-mediated interaction of the Eph-related receptor tyrosine kinase EphB3 and the ras-binding protein AF6 depends on the kinase activity of the receptor. Proc. Natl. Acad. Sci. U. S. A. 95:9779-9784.

58. Dodelet, V.C., Pazzagli, C., Zisch, A.H., Hauser, C.A., and Pasquale, E.B. 1999. A novel signaling intermediate, SHEP1, directly couples Eph receptors to R-Ras and Rap1A. J. Biol. Chem. 274:31941-31946.

59. Pandey, A., Duan, H., and Dixit, V.M. 1995. Characterization of a novel src-like adapter protein that associates with the Eck receptor tyrosine kinase. J. Biol. Chem. 270:19201-19204.

60. Cowan, C.A., and Henkemeyer,M.2001. TheSH2/SH3 adaptor Grb4 transduces B-ephrin reverse signals. Nature. 413:174-179.

61. Birgbauer, E., Cowan, C.A., Sretavan, D.W., and Henkemeyer, M. 2000. Kinase independent function of EphB receptors in retinal axon pathfinding to the optic disc from dorsal but not ventral retina. Development. 127:1231-1241.

62. Buchert, M., et al. 1999. The junction-associated protein AF-6 interacts and clusters with specific Eph receptor tyrosine kinases at specialized sites of cellcell contact in the brain. J. Cell Biol. 144:361-371.

63. Holland, S.J., et al. 1996. Bidirectional signaling through the EPH-family receptor Nuk and its transmembrane ligands. Nature. 383:722-725.

64. Palmer, A., et al. 2002. Ephrin B phosphorylation and reverse signaling: regulation by Src kinases and PTP-BL phosphatase. Mol. Cell. 9:725-737.

65. Xu, Z., Kwok-On, L., Zhou, H.-M., Lin, S.-C., and Ip, N.Y. 2003. Ephrin-B1 reverse signaling activates JNK through a novel mechanism that is independent of tyrosine phosphorylation. J. Biol. Chem. 278:24767-24775.

66. Schultz, J., Ponting, C.P., Hofmann, K., and Bork, P. 1997. SAM as a protein interaction domain involved in developmental regulation. Protein Sci. 6:249-253.

67. Thanos, C.D., Goodwill, K.E., and Bowie, J.U. 1999 Oligomeric structure of the human Ephb2 receptor SAM domain. Science. 283:833-836.

68. Stapleton, D., Balan, L., Pawson, T., and Sicheri, F. 1999. The crystal structure of an Eph receptor SAM domain reveals a mechanism for modular dimerization. Nat. Struct. Biol. 6:44-49.

69. Smalla, M., et al. 1999. Solution structure of the receptor tyrosine kinase EphB2 SAM domain and identification of two distinct homotypic interaction sites. Protein Sci. 8:1954-1961.

70. Wilkinson, D.G. 2000. Eph receptors and ephrins: regulators of guidance and assembly. Int. Rev. Cytol. 196:177-244.

71. Gale, N.W., and Yancopoulos, G.D. 1999. Growth factors acting via endothelial cell-specific receptor tyrosine kinases: VEGFs, angiopoietins, and ephrins in vascular development. Genes Dev. 13:1055-1066.

72. Prevost, N., Woulfe, D., Tanaka, T, and Brass, L.F. 2002. Interactions between Eph kinases and ephrins provide a mechanism to support platelet aggregation once cell-to-cell contact has occurred. Proc. Natl. Acad. Sci. U. S. A. 99:9219-9224.

73. Bertoni, A., et al. 2002. Relationships between Rap1b, affinity modulation of integrin $\alpha_{\mathrm{II}} \beta_{3}$, and the actin cytoskeleton. J. Biol. Chem. 277:25715-25721.

74. Chrzanowska-Wodnicka, M., Smyth, S.S., Schoenwaelder, S.M., Fischer, T.H., and White, G.C., 2nd. 2005. Rap1b is required for normal platelet function and hemostasis in mice [corrigendum 2005, 115:2296]. J. Clin. Invest. 115:680-687. doi:10.1172/ JCI200522793.

75. Prevost, N., et al. 2004. Signaling by ephrinB1 and Eph kinases in platelets promotes Rap1 activation, platelet adhesion, and aggregation via effector pathways that do not require phosphorylation of ephrinB1. Blood. 103:1348-1355.

76. Prevost, N., et al. 2005. Eph kinases and ephrins support thrombus growth and stability by regulating integrin outside-in signaling in platelets. Proc. Natl. Acad. Sci. U. S. A. 102:9820-9825.

77. Nanda, N., et al. 2005. Platelet endothelial aggregation receptor 1 (PEAR1), a novel epidermal growth factor repeat-containing transmembrane receptor, participates in platelet contact-induced activation. J. Biol. Chem. 280:24680-24689.

78. Coppinger, J.A., et al. 2004. Characterization of the proteins released from activated platelets leads to localization of novel platelet proteins in human atherosclerotic lesions. Blood. 103:2096-2104.

79. Bergmeier, W., et al. 2004. Tumor necrosis factoralpha-converting enzyme (ADAM17) mediates GPIbalpha shedding from platelets in vitro and in vivo. Circ. Res. 95:677-683.

80. Rabie, T., Strehl, A., Ludwig, A., and Nieswandt, B. 2005. Evidence for a role of ADAM17 (TACE) in the regulation of platelet glycoprotein V. J. Biol. Chem. 280:14462-14468.

81. Bergmeier, W., et al. 2004. GPVI down-regulation in murine platelets through metalloproteinase-dependent shedding. Thromb. Haemost. 91:951-958.

82. Stephens, G., et al. 2005. Platelet activation induces metalloproteinase-dependent GP VI cleavage to down-regulate platelet reactivity to collagen. Blood. 105:186-191.

83. Berger, G., Hartwell, D.W., and Wagner, D.D. 1998. P-selectin and platelet clearance. Blood. 92:4446-4452.

84. Henn, V., et al. 1998. CD40 ligand on activated platelets triggers an inflammatory reaction of endothelial cells. Nature. 391:591-594.

85. Henn, V., Steinbach, S., Buchner, K., Presek, P., and Kroczek, R.A. 2001. The inflammatory action of CD40 ligand (CD154) expressed on activated human platelets is temporally limited by coexpressed CD40. Blood. 98:1047-1054.

86. Hermann, A., Rauch, B.H., Braun, M. Schror, K., and Weber, A.A. 2001. Platelet CD40 ligand (CD40L): subcellular localization, regulation of expression, and inhibition by clopidogrel. Platelets. 12:74-82.

87. Locksley, R.M., Killeen, N., and Lenardo, M.J. 2001. The TNF and TNF receptor superfamilies: integrating mammalian biology. Cell. 104:487-501.

88. Andre, P., et al. 2002. CD40L stabilizes arterial thrombi by a $\beta 3$ integrin-dependent mechanism. Nat. Med. 8:247-252.

89. Inwald, D.P., McDowall, A., Peters, M.J., Callard, R.E., and Klein, N.J. 2003. CD40 is constitutively expressed on platelets and provides a novel mechanism for platelet activation. Circ. Res. 92:1041-1048.

90. Mach, F., Schonbeck, U., Sukhova, G.K., Akinson, E., and Libby, P. 1998. Reduction of atherosclerosis in mice by inhibition of CD40 signaling. Nature. 394:200-203.

91. May, A.E., et al. 2002. Engagement of glycoprotein IIb/IIIa on platelets upregulates CD40L and triggers CD40L-dependent matrix degradation by endothelial cells. Circulation. 106:2111-2117.

92. Danese, S., and Fiocchi, C. 2005. Platelet activation 
and the CD40/CD40 ligand pathway: mechanisms and implications for human disease. Crit. Rev. Immunol. 25:103-122.

93. Crow, A.R., Leytin, V., Starkey, A.F., Rand, M.L., and Lazarus, A.H. 2003. CD154 (CD40 ligand)deficient mice exhibit prolonged bleeding time and decreased shear-induced platelet aggregates. J. Thromb. Haemost. 1:850-852.

94. Tamagnone, L., and Comoglio, P.M. 2000. Signalling by semaphorin receptors: cell guidance and beyond. Trends Cell Biol. 10:377-383.

95. Yu, H.H., and Kolodkin, A.L. 1999. Semaphorin signaling: a little less per-plexin. Neuron. 22:11-14.

96. Love, C.A., et al. 2003. The ligand-binding face of the semaphorins revealed by the high-resolution crystal structure of SEMA4D. Nat. Struct. Biol. 10:843-848.

97. Kumanogoh, A., et al. 2000. Identification of CD72 as a lymphocyte receptor for the class IV semaphorin CD100: a novel mechanism for regulating B cell signaling. Immunity. 13:621-631.

98. Ishida, I., et al. 2003. Involvement of CD100, a lymphocyte semaphorin, in the activation of the human immune system via CD72: implications for the regulation of immune and inflammatory responses. Int. Immunol. 15:1027-1034.

99. Delaire, S., et al. 2001. Biological activity of soluble CD100. II. Soluble CD100, similarly to H-SemaIII, inhibits immune cell migration. J. Immunol. 166:4348-4354.

100.Wang, X., et al. 2001. Functional soluble CD100/ Sema4D released from activated lymphocytes: possible role in normal and pathologic immune responses. Blood. 97:3498-3504.
101. Shi, W., et al. 2000. The class IV semaphorin CD100 plays nonredundant roles in the immune system: defective $B$ and $T$ cell activation in CD100-deficient mice. Immunity. 13:633-642.

102.Basile, J.R., Barac, A., Zhu, T., Guan, K.L., and Gutkind, J.S. 2004. Class IV semaphorins promote angiogenesis by stimulating Rho-initiated pathways through plexin-B. Cancer Res. 64:5212-5224.

103. Conrotto, P., et al. 2005. Sema4D induces angiogenesis through Met recruitment by Plexin B1. Blood. 105:4321-4329.

104.Kumanogoh, A., and Kikutani, H. 2001. The CD100-CD72 interaction: a novel mechanism of immune regulation. Trends Immunol. 22:670-676.

105. Adachi, T. Flaswinkel, H., Yakura, H., Reth, M., and Tsubata, T. 1998. The B cell surface protein CD72 recruits the tyrosine phosphatase SHP-1 upon tyrosine phosphorylation. J. Immunol. 160:4662-4665.

106. Baba, T., et al. 2005. Dual regulation of BCR-mediated growth inhibition signaling by CD72. Eur. J. Immunol. 35:1634-1642.

107. Tamagnone, L., et al. 1999. Plexins are a large family of receptors for transmembrane, secreted, and GPIanchored semaphorins in vertebrates. Cell. 99:71-80.

108. Conrotto, P., Corso, S., Gamberini, S., Comoglio, P.M., and Giordano, S. 2004. Interplay between scatter factor receptors and B plexins controls invasive growth. Oncogene. 23:5131-5137.

109.Zhu, L., et al. 2003. Semaphorin 4D (CD100) is expressed on the surface of human platelets and proteolytically shed during platelet activation [abstract]. Blood. 102:292a.

110.Zhu, L., et al. 2005. Regulated shedding of sema4D from the platelet surface produces a bioactive sec- ond messenger in thrombotic disorders [abstract]. Blood. In press.

111. Melaragno, M.G., Fridell, Y.-W., and Berk, B.C. 1999. The Gas6/Axl system: a novel regulator of vascular cell function. Trends Cardiovasc. Med. 9:250-253.

112.Ishimoto, Y., and Nakano, T. 2000. Release of a product of growth arrest-specific gene 6 from rat platelets. FEBS Lett. 466:197-199.

113. Angelillo-Scherrer, A., et al. 2001. Deficiency or inhibition of Gas 6 causes platelet dysfunction and protects mice against thrombosis. Nat. Med. 7:215-221.

114.Stitt, T.N., et al. 1995. The anticoagulation factor protein and its relative, Gas6, are ligands for the Tyro3/Axl family of receptor tyrosine kinases. Cell. 80:661-670.

115.Varnum, B.C., et al. 1995. Axl receptor tyrosine kinase stimulated by the vitamin K-dependent protein encoded by the growth-arrest-specific gene 6 . Nature. 373:623-626.

116. Chen, C., et al. 2004. Mer receptor tyrosine kinase signaling participates in platelet function. Arterioscler. Thromb. Vasc. Biol. 24:1118-1123.

117. Angelillo-Scherrer, A., et al. 2005. Role of Gas6 receptors in platelet signaling during thrombus stabilization and implications for antithrombotic therapy. J. Clin. Invest. 115:237-246. doi:10.1172/ JCI200522079.

118.Gould, W.R., et al. 2005. Gas6 receptors Axl, Sky and Mer enhance platelet activation and regulate thrombotic responses. J. Thromb. Haemost. 3:733-741.

119. Balogh, I., Hafizi, S., Stenhoff, J., Hansson, K., and Dahlback, B. 2005. Analysis of Gas6 in human platelets and plasma. Arterioscler. Thromb. Vasc. Biol. 25:1280-1286. 